\title{
Rapport au savoir et éducation relative à l'environnement
}

Jacques Désautels

\section{(2) OpenEdition \\ Journals}

Édition électronique

URL : https://journals.openedition.org/ere/7315

DOI : $10.4000 /$ ere. 7315

ISSN : 2561-2271

Éditeur

Centr'ERE

Référence électronique

Jacques Désautels, «Rapport au savoir et éducation relative à l'environnement », Éducation relative à

l'environnement [En ligne], Volume 1 | 1999, mis en ligne le 15 septembre 1999, consulté le 28 mai

2021. URL : http://journals.openedition.org/ere/7315 ; DOI : https://doi.org/10.4000/ere.7315 


\title{
Rapport au savoir et éducation relative à l'environnement
}

\author{
Jacques Désautels
}

Tout système d'éducation est une manière

politique de maintenir ou de modifier

l'appropriation des discours, avec les savoirs et les pouvoirs qu'ils emportent avec eux. (Michel

Foucault, 1971, p. 46)

$1 \quad$ Il n'est plus possible de faire comme si la problématique du savoir pouvait être traitée isolément, en marge de considérations portant sur les contingences sociohistoriques inhérentes à sa production (Bourdieu, 1997), à sa distribution plus ou moins inégale parmi les membres d'une société et, de facto, à sa reproduction ${ }^{1}$ par des élèves en contexte scolaire. L'adoption d'une telle posture à l'égard de la problématique du savoir comporte une exigence de réflexivité qui oblige à repérer, au mieux, les taches aveugles de nos discours, leurs conditions de production, et ce, d'autant plus que toute forme d'éducation, selon la thèse exprimée par Foucault (1971) dans la citation en épigraphe, actualise nécessairement un projet sociopolitique. En effet, au sein de l'institution scolaire, le contrôle exercé sur le régime du discours fournit une légitimité à certains types d'énoncés au détriment de certains autres et contribue ainsi à la reproduction de la hiérarchie sociale des savoirs et, nécessairement, des pouvoirs qu'ils confèrent à ceux et celles qui en ont la maîtrise. En conséquence, il me semble donc pertinent que l'on s'interroge sur le type de rapport au savoir dit savant promu à l'école, en particulier dans le contexte de l'éducation relative à l'environnement (ERE). La stratégie que j'adopterai à cette fin sera en quelque sorte indirecte puisque je fais l'hypothèse que dans bon nombre de cas (Robottom et Hart, 1995) l'ERE en milieu scolaire se pratique dans le contexte général de l'éducation aux sciences ${ }^{2}$. Ainsi, après avoir procédé à un examen du type de rapport au savoir scientifique qui est promu en milieu scolaire, je tenterai d'en tirer quelques conséquences pour ce qui est de l'ERE. Mais, dans un premier temps, je m'efforcerai de cerner quelques-unes des prémisses qui orientent mon discours à ce sujet sous la forme de deux brèves propositions 
commentées, l'une que l'on pourrait qualifier d'idéologique et la seconde d'épistémologique, sachant que ces distinctions sont bien sûr analytiques.

\section{Éducation et citoyenneté}

2 Il me semble important de rappeler que l'une des finalités centrales de l'institution scolaire est d'assurer la formation de citoyennes et de citoyens avertis et critiques qui auront construit les potentiels de mobilisation (Rochex, 1995) et d'action (Morf, 1998) nécessaires à leur participation, ici et maintenant, au développement de la vie démocratique dans notre société ${ }^{3}$, entre autres, par un engagement dans les controverses sociotechniques qui la traversent, dont les controverses dites environnementales.

On remarquera que, dans cette proposition, l'accent est mis sur la citoyenneté plutôt que sur le leitmotiv qui imprègne le projet éducatif de l'école québécoise depuis quelques décennies ${ }^{4}(4)$, soit le développement de la personne. Par ailleurs, en spécifiant que c'est "ici et maintenant " que les élèves doivent agir dans la société (Désautels, 1998), je souligne que l'accès au statut de citoyen ou citoyenne constitue un apprentissage qui doit démarrer dès le début de la scolarisation des enfants. Enfin, j'y rappelle que les questions environnementales sont par définition des objets de controverses et que, par là, elles constituent l'un des lieux privilégiés de formation à la citoyenneté

\section{L'environnement existe-t-il ?}

4 La seconde proposition, de nature épistémologique, s'inscrit dans une perspective socioconstructiviste de la cognition. Elle porte notamment sur la signification que j'attribue au concept d'environnement.

5 Ce que collectivement nous nommons environnement, c'est chaque fois l'une des représentations parmi un nombre incalculable de possibles, construite et négociée, plus ou moins localement, entre des acteurs et des actrices sociaux, du point de vue particulier à partir duquel ils et elles effectuent des distinctions et des descriptions, et ce, afin de donner relief et sens à leurs expériences. Autrement dit, comme le soulignent Latour et coll. (1991), "Autant d'actions, autant de cultures, autant de sociétés, autant d'environnements - au pluriel ».

6 Cette proposition se démarque de celle en vertu de laquelle d'aucuns estiment qu'il est possible de raisonner sur une totalité (biosphère, cosmos, etc.) en la regardant en surplomb, comme si l'observateur était extérieur à ce qu'il décrit, ce que les anglophones nomment $A$ God's eye view of the world. Par ailleurs, elle réintroduit les observateurs et les observatrices avec leurs visions du monde, leurs valeurs et leurs grilles conceptuelles dans les processus sociaux de production des représentations qui, inévitablement, font intervenir des rapports de pouvoir. En effet, la légitimité de ce qui est dit, et donc de ce qui sera tenu comme un problème environnemental (Gilbert, 1998), dépend de la position des acteurs et actrices dans un champ particulier et de la position des champs dans la société. Enfin, en relativisant la hiérarchie sociale des savoirs, cette proposition ouvre sur d'autres possibles puisqu'il n'y a plus de position privilégiée pour dire l'environnement. En ce sens, la position dite scientifique au regard 
de ce qui sera considéré comme un problème environnemental, telle la position des divers types d'experts, en constitue une parmi tant d'autres. Mais qu'en est-il au juste de la question du rapport au savoir en contexte scolaire?

\section{Enseignement des sciences et rapport au savoir}

7 Toute forme d'appropriation des savoirs par les élèves implique la construction concomitante d'un rapport à ces savoirs et à ceux et celles qui en ont la maitrise. Ce rapport, comme nous le verrons, peut être plus ou moins émancipatoire, plus ou moins inhibant, voire aliénant. Mais que veut-on signifier par rapport au savoir? Au lieu de proposer une définition formelle de ce concept (Charlot, 1997), je vais plutôt en fournir une illustration par le biais d'une analyse d'un extrait de discours formulé par un élève au terme de ses études secondaires. Nous lui avions demandé s'il désirait ajouter des commentaires à la fin d'un entretien portant sur la nature et la production du savoir scientifique et il a énoncé le point de vue suivant.

J'ai de la misère à voir comment on peut faire [avec des choses qu'on ne voit pas]. Ils

[les professeurs] vont nous l'expliquer et on va comprendre comment ils [les scientifiques] ont pu voir ça. Ils vont arriver et dire :

la distance de la terre à la lune égale quelque chose... » je ne le sais pas par cœur. Mais comment ils ont fait pour mesurer ça ? Ils n'ont pas un galon à mesurer si long que ça ! J'ai bien de la misère avec ça [...] Et le gars [qui] a découvert ça : comment il a fait? Il est venu de même un matin et ça lui est arrivé ? C'est dur. Je vais comprendre un coup que ça va être expliqué, mais le gars à qui personne ne l'a jamais expliqué quand il l'a su, comment il a fait ? [...] Il devait être drôlement doué. Il faut certainement être doué, être intéressé, intelligent. Il y en a qui sont plus intelligents que d'autres. C'est comme la loi de la nature de tantôt : il y a des arbres qui grossissent et il y en a d'autres qui vont toujours rester tout le temps petits. (Désautels et Larochelle, 1989, p. 155)

Voir c'est croire, telle serait la posture épistémologique ${ }^{6}$ qui oriente ce type de discours. Les scientifiques produiraient ni plus ni moins une description littérale du monde, comme en témoignent la mention de la mesure de la distance Terre - Lune et, surtout, la référence à la capacité qui serait la leur de «voir» ce qui ne peut être vu par le commun des mortels (Larochelle, 1998). Dès lors le savoir scientifique est en quelque sorte réifié et ce qui est découvert, c'est-à-dire " sorti de sa cachette », comme le disait l'un de ses collègues, correspondrait aux états du monde. Dans ces circonstances, les sciences produiraient un discours véridique vis-à-vis duquel on ne peut que s'incliner. Ce type de rapport au savoir conduit à une forme de soumission à l'égard de la hiérarchie sociale des savoirs à l'intérieur de laquelle le savoir commun est parfaitement déconsidéré. Il est donc peu probable que cet étudiant puisse construire une position critique articulée vis-à-vis l'entreprise scientifique. Parallèlement, il est amené à se sous-estimer par rapport à ceux et celles qui sont ou deviendront des scientifiques : «il faut certainement être doué », ce qui inhibe toute velléité de sa part de participer à la production de ces savoirs puisque celle-ci ne serait pas accessible aux "petits arbres ». Enfin, cela peut le conduire à trouver normale ou "naturelle » la distribution actuelle des savoirs et des pouvoirs dans nos sociétés; il y aurait des personnes plus douées que d'autres et c'est pourquoi elles jouiraient de privilèges mérités. En somme, le type de rapport au savoir que les élèves établissent constitue une détermination importante dans le développement de leur potentiel de mobilisation et d'action nécessaire à leur engagement dans les controverses sociotechniques. 
Quel rapport avec l'ERE direz-vous? Eh bien, il suffit de remplacer dans l'extrait de discours qui précède la distance Terre - Lune par d'autres concepts (biosphère, écosystème, climat, couche d'ozone, effet de serre, etc.), pour imaginer que le même type de rapport au savoir peut être construit par les élèves si l'ERE devient une " matière scolaire » comme les autres et que, dans ce contexte, le seul concept légitime d'environnement est celui que les experts définissent. Dès lors, l'ERE ne contribuera pas davantage à la formation de citoyennes et de citoyens avertis et critiques que ne le fait actuellement l'enseignement des autres disciplines, à moins de supposer que l'ERE ait de telles vertus formatrices indépendantes de toute posture idéologique. Mais à quelles conditions une telle formation est-elle possible ? En voici quelques-unes qui, cela va de soi, mériteraient d'être explicites et débattues.

\section{Pour une désinstitutionnalisation de l'ERE}

Les activités pédagogiques doivent permettre aux élèves d'agir dans leur milieu social, ici et maintenant. En ce sens, le travail de Greenall Gough et Robottom (1993), entre autres, me semble exemplaire, de même que la stratégie de construction collective d'îlots de rationalité (Fourez, 1997) autour de problèmes dits environnementaux (épuration des eaux, élimination des déchets, etc.). Par ailleurs, les activités pédagogiques doivent aussi contribuer à relativiser la hiérarchie sociale des savoirs et le pouvoir de ceux qui les détiennent (écologistes, biologistes, etc.), et ainsi, de manière réflexive, d'une part, réhabiliter le savoir commun et d'autre part, redonner une place honorable aux autres traditions de connaissance (Descola et Pálsson, 1996) dans la négociation de ce qui sera tenu comme un enjeu environnemental. L'expérience actuellement conduite par Michael Roth en Colombie-Britannique montre que cela est tout à fait possible. Une étude d'impact reliée à la construction d'un club de golf près d'un marais et d'un ruisseau est réalisée conjointement par des élèves du premier cycle $d u$ secondaire et leurs enseignants et enseignantes, des personnes représentant la communauté amérindienne, des personnes représentant les aînés et des biologistes. Les apports des uns et des autres tant en ce qui a trait à la planification qu'à la réalisation des opérations font l'objet d'incessantes discussions et négociations, dans le cadre d'un réseau informatique et de discussions publiques. Par exemple, les élèves ont présenté à la mairie les résultats de leur recherche sur l'inventaire des crustacés le long du ruisseau qui se déverse dans la mer. En somme, comme il a été souligné ailleurs (Roth et McGinn, 1997) il est nécessaire de procéder à une désinstitutionnalisation de l'ERE si l'on souhaite former des personnes aptes à s'engager, comme le font déjà ces élèves, dans les controverses environnementales.

\section{BIBLIOGRAPHIE}

Bourdieu, P. (1997). Méditations pascaliennes. Paris : Éditions du Seuil.

Charlot, B. (1997). Du rapport au savoir. Paris : Éditions Anthropos. 
Conseil Supérieur de l'Éducation du Québec. (1998). Éduquer à la citoyenneté. Sainte-Foy : Le Conseil.

Désautels, J. (1998). Une éducation aux sciences pour l'action. Recherche en Soins Infirmiers, 52, $4-11$.

Désautels, J. et Larochelle, M. (1998). The epistemology of students : The 'thingified' nature of scientific knowledge. Dans B. Fraser et K.G. Tobin (dir.), International handbook of science education. Dordrecht : Kluwer Academic Publishers. P.115-126.

Désautels, J. et Larochelle, M. (1989). Qu'est-ce que le savoir scientifique ? Points de vue d'adolescents et d'adolescentes. Québec : Presses de l'Université Laval.

Descola, P. et Pálsson, G. (1996). Nature and society : Anthropological perspectives. New York : Routledge.

Foucault, M. (1971). L'ordre du discours. Paris : Éditions Gallimard.

Fourez, G. (1997). Scientific and technological literacy as a social practice. Social Studies of Science, 27, 903-936.

Gilbert, C. (1998). Le sens caché des risques collectifs. La Recherche, 307, 110-113.

Gouvernement du Québec. (1997). L'école tout un programme : énoncé de politique éducative. Québec : ministère de l'Éducation.

Greenall Gough, A. et Robottom, I. (1993). Towards a socially critical environmental edu-cation : Water quality studies in a coastal school. Journal of Curriculum Studies, 25(4), 301-316.

Larochelle, M. (1998). La tentation de la classification ou comment un apprentissage non réflexif des savoirs scientifiques peut donner lieu à un problème épistémologique. Recherche en Soins Infirmiers, 52, 72-80.

Latour, B., Schwartz, C. et Charvolin, F. (1991). Crises des environnements : défis aux sciences humaines. Futur Antérieur, 28-56.

Morf, A. (1998). An epistemology for didactics : Speculations on situating a concept. Dans M. Larochelle, N. Bednarz, et J. Garrison (dir.), Constructivism and education. New York : Cambridge University Press. p. 29-42.

Robottom, I. et Hart, P. (1995). Behaviorist EE research : Environmentalism as individua-lism. The Journal of Environmental Education, 26(2), 5-9.

Rochex, J-Y. (1995). Le sens de l'expérience scolaire. Paris : Presses Universitaires de France.

Roth, W.-M. et McGinn, M.K. (1997). Deinstitutionalising school science : Implications of a strong view of situated cognition. Research in Science Education, 27, 497-513.

\section{NOTES}

1. Le trait d'union indique ici qu'il ne s'agit pas d'un processus mécanique et déterministe de production de l'identique qui transformerait les actrices et les acteurs sociaux en "idiots culturels » incapables, en diverses occasions, de développer un quant-à-soi à l'égard du savoir canonique.

2. Il ne s'agit aucunement ici de nier la diversité des pratiques en ERE. Toutefois, on doit reconnaître qu'à l'école, pour le meilleur ou pour le pire, ce sont souvent dans les cours d'écologie et de biologie qu'ont lieu des activités ayant pour visée d'éduquer à l'envi rondement. 
3. L'énoncé de politique éducative du Gouvernement du Québec (1997) spécifie qu'il s'agit bien là d'une des missions de l'école à côté de l'instruction et de la qualification. Ce thème de réflexion vient d'ailleurs de faire l'objet d'un rapport substantiel du Conseil Supérieur de l'Éducation (1998).

4. Il importe de saisir que le fait d'opter pour la citoyenneté ne constitue pas une opposition au développement harmonieux de la personnalité des élèves ou encore de certains autres aspects de leur potentiel intellectuel. Toutefois, comme l'illustre bien le référendum qui a été tenu en Suisse en juin 1998 à propos des règles législatives qui encadrent la recherche en biotechnologie et, plus particulièrement des effets environnementaux anticipés de la propagation des plantes génétiquement modifiées, il devient impératif de préparer les élèves à jouer leur rôle de citoyen et de citoyenne, car ils et elles seront appelés à prendre position quant à de telles controverses.

5. Je suis parfaitement consciente que la notion de citoyenneté est une notion complexe qui n'a pas de signification universelle, pas plus d'ailleurs que celle de démocratie. En conséquence, le point de vue que je développe dans ce texte est en quelque sorte partial et orienté en fonction du lieu à partir duquel je parle. Toutefois, dans la mesure où l'on souhaite qu'une discussion sur ces sujets problématiques s'engagent entre les peuples, il est impératif que les uns et les autres clarifient au mieux leurs discours.

6. Les travaux récents dans le champ de l'éducation aux sciences tendent à montrer que le discours de cet élève ne constitue pas un cas d'exception (Désautels et Larochelle, 1998). Il n'y a pas raison de croire que les élèves s'appuieront sur d'autres prémisses épistémologiques pour penser les problèmes dits environnementaux.

\section{AUTEUR}

\section{JACQUES DÉSAUTELS}

Jacques Désautels est professeur de didactique des sciences à l'Université Laval (Québec) et s'intéresse principalement aux enjeux socioethniques et idéologiques liés à l'appropriation des savoirs scientifiques en contexte scolaire. Ses travaux sont reconnus internationalement comme en témoignent ses nombreuses publications dans le domaine. 